\title{
Existence and uniqueness of positive periodic solutions for a class of integral equations with parameters
}

\author{
by Shu-Gui Kang (Datong), Bao Shi (Yantai) and \\ Sui Sun Cheng (Hsinchu)
}

\begin{abstract}
Existence of periodic solutions of functional differential equations with parameters such as Nicholson's blowflies model call for the investigation of integral equations with parameters defined over spaces with periodic structures. In this paper, we study one such equation

$$
\phi(x)=\lambda \int_{[x, x+\omega] \cap \Omega} K(x, y) h(y) f(y, \phi(y-\tau(y))) d y, \quad x \in \Omega,
$$

by means of the proper value theory of operators in Banach spaces with cones. Existence, uniqueness and continuous dependence of proper solutions are established.
\end{abstract}

1. Introduction. Existence of solutions of differential equations is often established by means of fixed point theorems for integral equations. Such an approach naturally calls for the investigation of integral equations and operator equations. Recent investigations (see e.g. $[1,5-8]$ and the references cited in [7]) of the existence of periodic solutions of functional differential equations such as

$$
\phi^{\prime}(x)=-a(x) \phi(x)+f(\phi(x)), \quad x \in \mathbb{R},
$$

where $a=a(x)$ is a positive continuous $2 \pi$-periodic function defined on $\mathbb{R}$, show that fixed points techniques applied to integral equations of the form

$$
\phi(x)=\int_{x}^{x+2 \pi} K(x, y) f(\phi(y)) d y, \quad x \in \mathbb{R},
$$

where

$$
K(x, y)=\frac{\exp \int_{x}^{y} a(t) d t}{\exp \int_{0}^{2 \pi} a(t) d t-1}, \quad x, y \in \mathbb{R}
$$

2000 Mathematics Subject Classification: Primary 45M15; Secondary 45M05.

Key words and phrases: integral equations, positive periodic solution, cone, eigenvalue. 
can also lead to existence criteria for (1). These studies prompted us to investigate in [6] integral equations of the form

$$
\phi(x)=\int_{\Omega(x)} K(x, y) f(y, \phi(y-\tau(y))) d y
$$

where $\Omega(x)$ is a closed subset of $\mathbb{R}^{N}$ which depends on $x$ and has positive Lebesgue measure $\mu(\Omega(x))$, and derive the existence of periodic solutions by means of fixed point index theory.

Yet there are also functional differential equations in which "eigenvalues" are involved. For instance, we have Nicholson's well known blowflies model

$$
\eta^{\prime}(t)=-\delta \eta(t)+\lambda \eta(t-\tau) e^{-a \eta(t-\tau)}, \quad t \geq 0,
$$

where $\delta, a, \tau>0$ and $\lambda$ is a positive parameter (see e.g. [4]). Therefore, in this paper, we will study integral equations in which a parameter is also involved. By means of several existence theorems in monotone operator theory, we will establish some abstract existence, uniqueness and continuous dependence theorems for the corresponding periodic solutions. In the last section, we will return to Nicholson's blowflies model to see some applications of our results.

To be more precise, let us first recall some terminology from [6]. Let $\mathbb{R}^{N}$ be the $N$-dimensional Euclidean space endowed with componentwise ordering $\leq$. For any $u, v \in \mathbb{R}^{N}$, the "interval" $[u, v]$ is the set $\left\{x \in \mathbb{R}^{N} \mid u\right.$ $\leq x \leq v\}$. Let $\omega=\left(\omega_{1}, \ldots, \omega_{N}\right) \in \mathbb{R}^{N}$ with positive components and let $e^{(1)}=(1,0, \ldots, 0), \ldots, e^{(N)}=(0, \ldots, 0,1)$ be the standard orthonormal vectors in $\mathbb{R}^{N}$. Let $\Omega$ be a closed subset of $\mathbb{R}^{N}$ which has the following "periodic" structure: for each $x \in \Omega$,

$$
x+\omega_{i} e^{(i)} \in \Omega,
$$

and for each pair $y, z \in \Omega$,

$$
\mu([y, y+\omega] \cap \Omega)=\mu([z, z+\omega] \cap \Omega)>0 .
$$

For convenience, we set

$$
\Omega(x)=[x, x+\omega] \cap \Omega .
$$

An example of such a set can be found in [6].

We will be concerned with integral equations of the form

$$
\phi(x)=\lambda \int_{[x, x+\omega] \cap \Omega} K(x, y) h(y) f(y, \phi(y-\tau(y))) d y, \quad x \in \Omega,
$$

where the functions $K, h, f, \tau$ and parameter $\lambda$ satisfy the following basic conditions: 
- $K \in C(\Omega \times \Omega,(0, \infty))$ and $K\left(x+\omega_{i} e^{(i)}, y+\omega_{i} e^{(i)}\right)=K(x, y)$ for any $(x, y) \in \Omega \times \Omega$ and $i \in\{1, \ldots, N\}$, and $K$ is uniformly continuous $\left({ }^{1}\right)$ on $\Omega \times \Omega$,

- $h \in C(\Omega,(0, \infty))$ and $h\left(x+\omega_{i} e^{(i)}\right)=h(x)$ for any $x \in \Omega$ and $i \in$ $\{1, \ldots, N\}$,

- $f \in C(\Omega \times[0, \infty),[0, \infty))$ and $f\left(x+\omega_{i} e^{(i)}, u\right)=f(x, u)$ for any $x \in \Omega$ and $i \in\{1, \ldots, N\}$,

- $\tau: \Omega \rightarrow \Omega$ is continuous and $\tau\left(x+\omega_{i} e^{(i)}\right)=\tau(x)$ for any $x \in \Omega$ and $i \in\{1, \ldots, N\}$,

- $\lambda>0$.

Our main concern will be the existence and uniqueness of positive periodic solutions of our equation (3). More precisely, we will look for solutions in the set of all real continuous functions of the form $\phi: \Omega \rightarrow \mathbb{R}$ such that

$$
\phi\left(x+\omega_{i} e^{(i)}\right)=\phi(x), \quad x \in \Omega, i \in\{1, \ldots, N\} .
$$

This set will be denoted by $C_{\omega}(\Omega)$. We say that a function $\phi \in C_{\omega}(\Omega)$ is an $\omega$-periodic solution of (3) associated with the parameter $\lambda \in(0, \infty)$ if substitution of $\phi$ and $\lambda$ into (3) turns it into an identity for every $x \in \Omega$. Since (3) may be interpreted as an equilibrium population distribution model, it is of interest to find parameters $\lambda$ and the associated "positive" periodic solutions of (3). We will show that for a (nonnegative) function $f$ with appropriate sublinear and/or superlinear behaviors, positive periodic solutions exist with associated parameters that lie in an interval, and that such solutions are unique if $f$ has additional monotonicity and convexity properties.

Let $E$ be a real Banach space, and $P$ a cone in $E$. The semi-order induced by the cone $P$ is denoted by " $\leq$ ": $x \leq y$ if and only if $y-x \in P$. Note that when endowed with the usual linear and ordering structure as well as the norm $\|\phi\|=\max _{z \in \Omega(x), x \in \Omega}|\phi(z)|, C_{\omega}(\Omega)$ is a normed ordered linear space with the cone $P=\left\{\phi \in C_{\omega}(\Omega): \phi(x) \geq 0\right.$ for all $\left.x \in \Omega\right\}$.

Definition 1 ([2]). A cone in a real Banach space is said to be solid if it has nonempty interior.

Definition 2 ([2]). Let $P$ be a solid cone in a real Banach space $E$ and denote by $P^{\circ}$ the interior of $P$. An operator $A: P^{\circ} \rightarrow P^{\circ}$ is called $\alpha$-concave (respectively $-\alpha$-convex) if $A(t \hbar) \geq t^{\alpha} A \hbar$ (respectively $A(t \hbar) \leq t^{-\alpha} A \hbar$ ) for any $\hbar \in P^{\circ}$ and $0<t<1$, where $0 \leq \alpha<1$. The operator $A$ is increasing (respectively decreasing) if $\hbar_{1}, \hbar_{2} \in P^{\circ}$ and $\hbar_{1} \leq \hbar_{2}$ imply $A \hbar_{1} \leq$ $A \hbar_{2}$ (respectively $A \hbar_{1} \geq A \hbar_{2}$ ); finally, $A$ is strongly increasing (respectively strongly decreasing) if $\hbar_{1}, \hbar_{2} \in P^{\circ}$ and $\hbar_{1}<\hbar_{2}$ imply $A \hbar_{2}-A \hbar_{1} \in P^{\circ}$

$\left({ }^{1}\right)$ This assumption can be relaxed. Indeed, it suffices to assume that for any $\varepsilon>0$, there exists $\delta>0$, which does not depend on $y$, such that $\left|K\left(x_{1}, y\right)-K\left(x_{2}, y\right)\right|<\varepsilon$ for all $x_{1}, x_{2} \in \Omega$ that satisfy $\left|x_{1}-x_{2}\right|<\delta$. 
(respectively $A \hbar_{1}-A \hbar_{2} \in P^{\circ}$ ). In case the equation $A \hbar=\lambda \hbar$, where $\lambda$ is a real parameter, has a unique solution in $P^{\circ}$, it will be denoted by $\hbar_{\lambda}$. Then $\hbar_{\lambda}$ is said to be strongly increasing (respectively strongly decreasing) in $\lambda$ if $\lambda_{1}>\lambda_{2}$ implies $\hbar_{\lambda_{1}}-\hbar_{\lambda_{2}} \in P^{\circ}$ (respectively $\left.\hbar_{\lambda_{2}}-\hbar_{\lambda_{1}} \in P^{\circ}\right)$, which is denoted by $\hbar_{\lambda_{1}} \gg \hbar_{\lambda_{2}}\left(\hbar_{\lambda_{2}} \gg \hbar_{\lambda_{1}}\right)$.

Lemma A ([3]). Suppose D is an open subset of an infinite-dimensional real Banach space $E$ with cone $P$. Suppose further that the null element $\theta$ of $E$ belongs to $D$. Let $\partial D$ be the boundary of $D$. If the operator $\Gamma: P \cap \bar{D} \rightarrow P$ is completely continuous with $\Gamma \theta=\theta$ and satisfies

$$
\inf _{\hbar \in P \cap \partial D}\|\Gamma \hbar\|>0
$$

then $\Gamma$ has a proper element in $P \cap \partial D$ associated with a positive eigenvalue. That is, there exist $\hbar_{0} \in P \cap \partial D$ and $\mu_{0}>0$ such that $\Gamma \hbar_{0}=\mu_{0} \hbar_{0}$.

Lemma B ([2]). Suppose $P$ is a normal solid cone of a real Banach space and $A: P^{\circ} \rightarrow P^{\circ}$ is an $\alpha$-concave increasing (or $\alpha$-convex decreasing) operator. Then $A$ has a unique fixed point in $P^{\circ}$.

2. Main results. Suppose that

$$
0<m \leq K(x, y) \leq M<\infty \text { for } x, y \in \Omega(t) \text { and } t \in \Omega,
$$

and

$$
M \neq m \text {. }
$$

Then

$$
1 \geq \frac{K(x, y)}{\sup _{x, y \in \Omega(t), t \in \Omega} K(x, y)} \geq \frac{\inf _{x, y \in \Omega(t), t \in \Omega} K(x, y)}{\sup _{x, y \in \Omega(t), t \in \Omega} K(x, y)} \geq \frac{m}{M}=\sigma \in(0,1) .
$$

Let $P_{1}=\left\{\phi \in C_{\omega}(\Omega): \phi(x) \geq \sigma\|\phi\|\right.$ for all $\left.x \in \Omega\right\}$. Then $P_{1}$ is a cone in $C_{\omega}(\Omega)$. Define an operator $F: C_{\omega}(\Omega) \rightarrow C_{\omega}(\Omega)$ by

$$
(F u)(x)=\int_{\Omega(x)} K(x, y) h(y) f(y, u(y-\tau(y))) d y, \quad x \in \Omega .
$$

Then for $x \in \Omega$,

$$
(F u)(x) \leq M \int_{\Omega(x)} h(y) f(y, u(y-\tau(y))) d y
$$

and

$$
(F u)(x) \geq m \int_{\Omega(x)} h(y) f(y, u(y-\tau(y))) d y \geq \sigma\|F u\| .
$$

Lemma 1. Suppose (4) holds. Then $F P_{1} \subset P_{1}$. 
Next, we need to impose an additional condition on the function $f$ and some additional notation. The additional condition is that

$$
f: \Omega \times[0, \infty) \rightarrow[0, \infty) \text { is continuous with } f(s, 0)=0 \text { for } s \in \Omega .
$$

We also set

$$
m(r)=\inf _{\sigma r \leq u \leq r, s \in \Omega(x), x \in \Omega} f(s, u) \quad \text { and } \quad B=\int_{\Omega(x)} h(s) d s,
$$

where, in view of the assumptions on $h$ and $\Omega$, we see that $B$ is a constant and $B>0$.

Theorem 1. Suppose that (4) and (8) hold and that there exist $l_{2}>$ $l_{1}>0$ and $\zeta>0$ such that $l_{1} u<f(y, u)<l_{2} u$ for all $y \in \Omega$ and all $u \geq \zeta$. Then there exist positive numbers $R_{0}, \lambda_{1}$ and $\lambda_{2}$ such that for any $r>R_{0}$, (3) has a positive $\omega$-periodic solution $u_{r}^{*}$ associated with some $\lambda^{*} \in\left[\lambda_{1}, \lambda_{2}\right]$ and $\left\|u_{r}^{*}\right\|=r$.

Proof. Note first that (3) has a positive $\omega$-periodic solution $u_{r}^{*}$ associated with $\lambda^{*}>0$ if and only if the operator $F$ has a proper element $u_{r}^{*}$ associated with the eigenvalue $1 / \lambda^{*}>0$. Let $R_{0}=\sigma^{-1} \zeta$ and

$$
G_{r}=\left\{u \in C_{\omega}(\Omega):\|u\|<r\right\},
$$

where $r>R_{0}$. Then $G_{r}$ is a bounded open subset of the Banach space $C_{\omega}(\Omega)$ and $\theta \in G_{r}$. In view of Lemma 1 and the properties of $K, h$ and $f$, we may show that $F: P_{1} \cap \bar{G}_{r} \rightarrow P_{1}$ is completely continuous with $F \theta=\theta$. Further,

$$
\begin{aligned}
(F u)(x) & =\int_{\Omega(x)} K(x, y) h(y) f(y, u(y-\tau(y))) d y \\
& \geq m \int_{\Omega(x)} h(y) f(y, u(y-\tau(y))) d y \\
& \geq l_{1} m \sigma\|u\| \int_{\Omega(x)} h(y) d y=l_{1} m \sigma B r>0
\end{aligned}
$$

for any $r>R_{0}$ and $u \in P_{1} \cap \partial G_{r}$. So we have

$$
\inf _{u \in P_{1} \cap \partial G_{r}}\|F u\| \geq l_{1} m \sigma B r>0 .
$$

By Lemma A, for any $r>R_{0}$, the operator $F$ has a proper element $u_{r}^{*} \in P_{1}$ associated with an eigenvalue $\mu^{*}>0$, and $u_{r}^{*}$ satisfies $\left\|u_{r}^{*}\right\|=r$. Let $\lambda^{*}=$ $1 / \mu^{*}$. Then $(3)$ has a positive $\omega$-periodic solution $u_{r}^{*}$ associated with $\lambda^{*}$. Thus, for any $r>R_{0}$, there exists a positive $\omega$-periodic solution $u_{r}^{*} \in P_{1} \cap$ $\partial G_{r}$ associated with $\lambda^{*}>0$. That is,

$$
u_{r}^{*}(x)=\lambda^{*} \int_{\Omega(x)} K(x, y) h(y) f\left(y, u_{r}^{*}(y-\tau(y))\right) d y \quad \text { with }\left\|u_{r}^{*}\right\|=r .
$$


Then we have

$$
\begin{aligned}
u_{r}^{*}(x) & \leq \lambda^{*} M \int_{\Omega(x)} h(y) f\left(y, u_{r}^{*}(y-\tau(y))\right) d y \\
& \leq \lambda^{*} M l_{2} r \int_{\Omega(x)} h(y) d y \leq \lambda^{*} M l_{2} B r
\end{aligned}
$$

and so

$$
\left\|u_{r}^{*}\right\|=r \leq \lambda^{*} M l_{2} B r
$$

which means that

$$
\lambda^{*} \geq \frac{1}{M l_{2} B}=\lambda_{1}
$$

Meanwhile,

$$
\begin{aligned}
u_{r}^{*}(x) & \geq \lambda^{*} m \int_{\Omega(x)} h(y) f\left(y, u_{r}^{*}(y-\tau(y))\right) d y \\
& \geq \lambda^{*} m l_{1} \sigma\left\|u_{r}^{*}\right\| \int_{\Omega(x)} h(y) d y=\lambda^{*} m l_{1} B \sigma r
\end{aligned}
$$

thus

$$
\left\|u_{r}^{*}\right\|=r \geq \lambda^{*} m l_{1} B \sigma r
$$

so we have

$$
\lambda^{*} \leq \frac{1}{m l_{1} B \sigma}=\lambda_{2} \quad \text { and } \quad \lambda_{1}<\lambda_{2} .
$$

Consequently, $\lambda^{*} \in\left[\lambda_{1}, \lambda_{2}\right]$. The proof is complete.

TheOREM 2. Suppose that (4) and (8) hold and that there exist $l_{1}>0$ and $\zeta>0$ such that $f(y, u)>l_{1} u$ for $u \geq \zeta$ and all $y \in \Omega$. Then there exist positive numbers $R_{0}$ and $\bar{\lambda}$ such that for any $r>R_{0}$, (3) has a positive $\omega$-periodic solution $\bar{u}^{*}$ associated with some $\bar{\lambda}^{*} \in(0, \bar{\lambda}]$ and $\left\|\bar{u}^{*}\right\|=r$.

The proof is similar to the proof of Theorem 1 and so is omitted.

TheOREm 3. Suppose that (4) and (8) hold and there exist $r_{0}>0$ and constants $c_{2}>c_{1}>0$ such that $c_{1} u<f(y, u)<c_{2} u$ for $0<u<r_{0}$ and all $y \in \Omega$. Then there exist positive numbers $r_{0}, \widehat{\lambda}_{1}$ and $\widehat{\lambda}_{2}$ such that for any $r \in\left(0, r_{0}\right),(3)$ has a positive $\omega$-periodic solution $\widehat{u}_{r}$ associated with some $\widehat{\lambda}^{*} \in\left[\widehat{\lambda}_{1}, \widehat{\lambda}_{2}\right]$ and $\left\|\widehat{u}_{r}\right\|=r$.

Proof. Define

$$
V_{r}=\left\{u \in C_{\omega}(\Omega):\|u\|<r\right\},
$$

where $0<r \leq r_{0}$. Then $V_{r}$ is a bounded open subset of the Banach space $C_{\omega}(\Omega)$ and $\theta \in V_{r}$. In view of Lemma 1 and properties of $K, f$ and $h$, we 
may show that $F: P_{1} \cap \bar{V}_{r} \rightarrow P_{1}$ is completely continuous with $F \theta=\theta$ and

$$
\begin{aligned}
F u(x) & =\int_{\Omega(x)} K(x, y) h(y) f(y, u(y-\tau(y))) d y \\
& \geq m \int_{\Omega(x)} h(y) f(y, u(y-\tau(y))) d y \\
& \geq c_{1} m \sigma\|u\| \int_{\Omega(x)} h(y) d y=c_{1} m \sigma B r>0
\end{aligned}
$$

for any $0<r<r_{0}$ and $u \in P_{1} \cap \partial V_{r}$. Thus

$$
\inf _{u \in P_{1} \cap \partial V_{r}}\|F u\| \geq c_{1} m \sigma B r>0 .
$$

By Lemma A, for any $0<r<r_{0}$, the operator $F$ has a proper element $\widehat{u}_{r} \in P_{1}$ associated with an eigenvalue $\widehat{\mu}>0$ and $\widehat{u}_{r}$ satisfies $\left\|\widehat{u}_{r}\right\|=r$. Letting $\widehat{\lambda}^{*}=1 / \widehat{\mu}$, we may then follow the last part of the proof of Theorem 1 to complete our proof.

TheOREM 4. Suppose that (4) and (8) hold and that there exist $c_{1}>0$ and $r_{0}>0$ such that $f(y, u)>c_{1} u$ for $0<u<r_{0}$ and all $y \in \Omega$. Then there exist positive numbers $r_{0}$ and $\widehat{\lambda}_{*}$ such that for any $r \in\left(0, r_{0}\right)$, (3) has a positive $\omega$-periodic solution $\widehat{u}_{r}$ associated with some $\widehat{\lambda} \in\left(0, \widehat{\lambda}_{*}\right]$ and $\left\|\widehat{u}_{r}\right\|=r$.

The proof is similar to that of Theorem 3; we omit it here.

Theorem 5. Suppose that (4) and (8) hold and that there exist $\widetilde{r}>0$ and $c_{\widetilde{r}}>0$ such that $m(\widetilde{r}) \geq c_{\widetilde{r}}>0$. Then there is a positive number $\widehat{\lambda}_{*}$ such that (3) has a positive $\omega$-periodic solution $\widehat{u}_{\widetilde{r}}$ associated with some $\widehat{\lambda} \in\left(0, \widehat{\lambda}_{*}\right]$ and $\left\|\widehat{u}_{\widetilde{r}}\right\|=\widetilde{r}$.

Proof. Indeed, let

$$
V_{\widetilde{r}}=\left\{u \in C_{\omega}(\Omega):\|u\|<\widetilde{r}\right\} .
$$

Then $V_{\widetilde{r}}$ is a bounded open subset of the Banach space $C_{\omega}(\Omega)$ and $\theta \in V_{\widetilde{r}}$, and $F: P_{1} \cap \bar{V}_{\widetilde{r}} \rightarrow P_{1}$ is completely continuous with $F \theta=\theta$. Furthermore,

$$
\inf _{u \in P_{1} \cap \partial V_{\widetilde{r}}}\|F u\| \geq c_{\widetilde{r}} m B>0 .
$$

From Lemma A, for $\widetilde{r}$, the operator $F$ has a proper element $\widehat{u}_{\widetilde{r}} \in P_{1} \cap \partial V_{\widetilde{r}}$ associated with an eigenvalue $\widetilde{\mu}^{*}>0$. Let $\widehat{\lambda}=1 / \widehat{\mu}^{*}$. Then (3) has a positive $\omega$-periodic solution $\widehat{u}_{\widetilde{r}} \in P_{1} \cap \partial V_{\widetilde{r}}$ associated with $\widehat{\lambda}>0$. That is,

$$
\widehat{u}_{\widetilde{r}}(x)=\widehat{\lambda} \int_{\Omega(x)} K(x, y) h(y) f\left(y, \widehat{u}_{\widetilde{r}}(y-\tau(y))\right) d y \quad \text { with }\left\|\widehat{u}_{\widetilde{r}}\right\|=\widetilde{r} .
$$


Thus we have

$$
\begin{aligned}
\widehat{u}_{\widetilde{r}}(x) & =\widehat{\lambda} \int_{\Omega(x)} K(x, y) h(y) f\left(y, \widehat{u}_{\widetilde{r}}(y-\tau(y))\right) d y \\
& \geq \widehat{\lambda} m c_{\widetilde{r}} \int_{\Omega(x)} h(y) d y=\widehat{\lambda} m c_{\widetilde{r}} B
\end{aligned}
$$

so that

$$
\left\|\widehat{u}_{\widetilde{r}}\right\|=\widetilde{r} \geq \widehat{\lambda} m c_{\widetilde{r}} B
$$

and hence

$$
\widehat{\lambda} \leq \frac{\widetilde{r}}{m c_{\widetilde{r}} B}=\widehat{\lambda}_{*}
$$

The proof is complete.

\section{Uniqueness and continuous dependence in concave increasing} case. So far we have not been able to prove uniqueness for the solutions $u_{\lambda}$ found in the previous results. However, when $f$ is monotone and concave in the sense defined below, uniqueness can be established together with continuous dependence on the parameters $\lambda$.

We note that $P=\left\{u \in C_{\omega}(\Omega): u(x) \geq 0\right.$ for all $\left.x \in \Omega\right\}$ is a normal solid cone of $C_{\omega}(\Omega)$ and its interior is $P^{\circ}=\left\{u \in C_{\omega}(\Omega): u(x)>0\right.$ for all $\left.x \in \Omega\right\}$.

Theorem 6. Suppose that $f: \Omega \times[0, \infty) \rightarrow[0, \infty)$ is a nondecreasing function in the second variable with $f(s, u)>0$ for $u>0$ and any $s \in \Omega$, and satisfies $f(s, t u) \geq t^{\alpha} f(s, u)$ for any $0<t<1$, where $0 \leq \alpha<1$. Then for any $\lambda>0,(3)$ has a unique positive $\omega$-periodic solution $u_{\lambda}$, and $u_{\lambda}$ has the following properties:

(i) $u_{\lambda}$ is strongly increasing in $\lambda$ on the cone $P$, that is, $\lambda_{1}>\lambda_{2}>0$ implies $u_{\lambda_{1}} \gg u_{\lambda_{2}}$

(ii) $\lim _{\lambda \rightarrow 0^{+}}\left\|u_{\lambda}\right\|=0$ and $\lim _{\lambda \rightarrow \infty}\left\|u_{\lambda}\right\|=\infty$;

(iii) $u_{\lambda}$ is continuous with respect to $\lambda$, that is, $\lim _{\lambda \rightarrow \lambda_{0}}\left\|u_{\lambda}-u_{\lambda_{0}}\right\|=0$ for any $\lambda_{0}>0$.

Proof. Let $\Phi=\lambda F$ for any $\lambda>0$. In view of (5)-(7), we have $\Phi P \subset P$. Because $h(y)>0, f(y, u)>0$ for $u>0$ and any $y \in \Omega$, and $K(x, y)>0$, we see that $\Phi: P^{\circ} \rightarrow P^{\circ}$. We assert that $\Phi: P^{\circ} \rightarrow P^{\circ}$ is an $\alpha$-concave increasing operator. Indeed, for any $0<t<1$,

$$
\begin{aligned}
\Phi(t u)(x) & =\lambda \int_{\Omega(x)} K(x, y) h(y) f(y, t u(y-\tau(y))) d y \\
& \geq \lambda t^{\alpha} \int_{\Omega(x)} K(x, y) h(y) f(y, u(y-\tau(y))) d y=t^{\alpha} \Phi(u)(x),
\end{aligned}
$$


where $0 \leq \alpha<1$. Since $f(y, u)$ is nondecreasing on $u$, we see that

$$
\begin{aligned}
\left(\Phi u_{1}\right)(x) & =\lambda \int_{\Omega(x)} K(x, y) h(y) f\left(y, u_{1}(y-\tau(y))\right) d y \\
& \leq \lambda \int_{\Omega(x)} K(x, y) h(y) f\left(y, u_{2}(y-\tau(y))\right) d y=\left(\Phi u_{2}\right)(x)
\end{aligned}
$$

for $u_{1}, u_{2} \in C_{\omega}(\Omega)$ such that $u_{1} \leq u_{2}$. By Lemma B, $\Phi$ has a unique fixed point $u_{\lambda} \in P^{\circ}$.

Next, we turn to the proof of (i)-(iii). Assume $0<\lambda_{2}<\lambda_{1}$. Then $u_{\lambda_{1}} \geq u_{\lambda_{2}}$. Indeed, define

$$
\bar{\eta}=\sup \left\{\widetilde{\eta}: u_{\lambda_{1}} \geq \widetilde{\eta} u_{\lambda_{2}}\right\} .
$$

We assert $\bar{\eta} \geq 1$. If this is not true, then $0<\bar{\eta}<1$, and

$$
\frac{1}{\lambda_{1}} u_{\lambda_{1}}=F u_{\lambda_{1}} \geq F\left(\bar{\eta} u_{\lambda_{2}}\right) \geq \bar{\eta}^{\alpha} F u_{\lambda_{2}}=\frac{1}{\lambda_{2}} \bar{\eta}^{\alpha} u_{\eta_{2}},
$$

which implies

$$
u_{\lambda_{1}} \geq \bar{\eta}^{\alpha} \frac{\lambda_{1}}{\lambda_{2}} u_{\lambda_{2}}
$$

but since $\bar{\eta}^{\alpha} \frac{\lambda_{1}}{\lambda_{2}}>\bar{\eta}$, this is a contradiction to (10), so $\bar{\eta} \geq 1$.

From the discussion above, we have

$$
u_{\lambda_{1}}=\lambda_{1} F u_{\lambda_{1}} \geq \lambda_{1} F u_{\lambda_{2}}=\frac{\lambda_{1}}{\lambda_{2}} u_{\lambda_{2}} \gg u_{\lambda_{2}} .
$$

Thus, $u_{\lambda}$ is strongly increasing in $\lambda$.

Set $\lambda_{2}=\lambda$ and fix $\lambda_{1}$ in (11). We have $u_{\lambda_{1}} \geq\left(\lambda_{1} / \lambda\right) u_{\lambda}$ for $\lambda_{1}>\lambda$, and

$$
\left\|u_{\lambda}\right\| \leq \frac{\lambda N_{1}}{\lambda_{1}}\left\|u_{\lambda_{1}}\right\|
$$

where $N_{1}>0$ is a constant. We thus have $\lim _{\lambda \rightarrow 0^{+}}\left\|u_{\lambda}\right\|=0$. Let $\lambda_{1}=\lambda$, and fix $\lambda_{2}$. From (11) and the normality of $P$, we have $\lim _{\lambda \rightarrow \infty}\left\|u_{\lambda}\right\|=\infty$.

Next, we show the continuity of $u_{\lambda}$ with respect to $\lambda$. For any $\lambda_{0}>0$, by (i),

$$
u_{\lambda} \ll u_{\lambda_{0}} \quad \text { for any } \lambda_{0}>\lambda \text {. }
$$

Set $l_{\eta}=\sup \left\{\zeta>0: u_{\lambda} \geq \zeta u_{\lambda_{0}}, \lambda_{0}>\lambda\right\}$. Obviously, $0<l_{\eta}<1$ and $u_{\lambda} \geq l_{\eta} u_{\lambda_{0}}$. Thus, we have

$$
\frac{1}{\lambda} u_{\lambda}=F u_{\lambda} \geq F\left(l_{\eta} u_{\lambda_{0}}\right) \geq l_{\eta}^{\alpha} F u_{\lambda_{0}}=\frac{1}{\lambda_{0}} l_{\eta}^{\alpha} u_{\lambda_{0}},
$$

and

$$
u_{\lambda} \geq \frac{\lambda}{\lambda_{0}} l_{\eta}^{\alpha} u_{\lambda_{0}}
$$


By the definition of $l_{\eta}$,

$$
\frac{\lambda}{\lambda_{0}} l_{\eta}^{\alpha} \leq l_{\eta} \quad \text { or } \quad l_{\eta} \geq\left(\frac{\lambda}{\lambda_{0}}\right)^{1 /(1-\alpha)},
$$

and we have

$$
u_{\lambda} \geq\left(\frac{\lambda}{\lambda_{0}}\right)^{1 /(1-\alpha)} u_{\lambda_{0}} \quad \text { for any } \lambda_{0}>\lambda .
$$

Since $P$ is a normal cone, by (13) and (14), we see that

$$
\left\|u_{\lambda_{0}}-u_{\lambda}\right\| \leq N_{2}\left[1-\left(\frac{\lambda}{\lambda_{0}}\right)^{1 /(1-\alpha)}\right]\left\|u_{\lambda_{0}}\right\| \rightarrow 0, \quad \lambda \rightarrow \lambda_{0}^{-},
$$

where $N_{2}$ is a positive constant. In the same way, we obtain $\left\|u_{\lambda}-u_{\lambda_{0}}\right\| \rightarrow 0$ as $\lambda \rightarrow \lambda_{0}^{+}$. The proof is complete.

4. An example. As an example, we consider the existence of positive $T$-periodic solutions for the equation

$$
\eta^{\prime}(t)=-\delta(t) \eta(t)+\lambda h(t) \eta(t-\tau) e^{-a \eta(t-\tau)},
$$

where $\delta=\delta(t)$ and $h=h(t)$ are positive continuous $T$-periodic functions defined on $\mathbb{R}^{+}=[0, \infty)$, and $\tau, a, \lambda \in(0, \infty)$. Note that if we take $\delta(t)=$ $\delta>0$ and $h(t)=1$, then (15) is the Nicholson blowflies model in [4].

We may check that a $T$-periodic solution of

$$
\eta(t)=\lambda \int_{t}^{t+T} H(t, s) h(s) f(\eta(s-\tau)) d s, \quad t \in \mathbb{R},
$$

where $f(u)=u e^{-a u}$ and

$$
H(t, s)=\frac{\exp \int_{t}^{s} \delta(u) d u}{\exp \int_{0}^{T} \delta(u) d u-1}, \quad s \in[t, t+T], t \in \mathbb{R},
$$

is also a $T$-periodic solution of (15).

We can verify that

- $H \in C(\mathbb{R} \times \mathbb{R},(0,+\infty)), H(x+T, y+T)=H(x, y)$ for any $(x, y) \in \mathbb{R} \times \mathbb{R}$ and $H$ is uniformly continuous on $\mathbb{R} \times \mathbb{R}$,

- $h \in C(\mathbb{R},(0, \infty))$ and $h(x+T)=h(x)$ for any $x \in \mathbb{R}$,

- $f \in C\left(\mathbb{R}^{+}, \mathbb{R}^{+}\right)$,

and

$$
\frac{\min _{t \in[0, T], s \in[t, t+T]} H(t, s)}{\max _{t \in[0, T], s \in[t, t+T]} H(t, s)}=\frac{m^{\prime}}{M^{\prime}}=e^{-\int_{0}^{T} \delta(u) d u}=\sigma^{\prime} \in(0,1) .
$$


Set

$$
(\Phi \eta)(t)=\int_{t}^{t+T} H(t, s) h(s) f(\eta(s-\tau)) d s .
$$

Let $C_{T}\left(\mathbb{R}^{+}\right)$be the set of all real $T$-periodic continuous functions defined on $\mathbb{R}^{+}$, endowed with the usual linear structure as well as the norm

$$
\|y\|=\sup _{t \in[0, T]}|y(t)| \text {. }
$$

Then $C_{T}\left(\mathbb{R}^{+}\right)$is a Banach space. Define a cone in $C_{T}\left(\mathbb{R}^{+}\right)$by

$$
P_{2}=\left\{y \in C_{T}\left(\mathbb{R}^{+}\right): y(t) \geq \sigma\|y\| \text { for all } t \in \mathbb{R}\right\} .
$$

If the operator $\Phi$ has a positive proper element $\eta \in C_{T}\left(\mathbb{R}^{+}\right)$associated with the eigenvalue $\mu$, that is,

$$
\Phi \eta=\mu \eta
$$

then (15) has a positive $T$-periodic solution $\eta(t)$. The function $f(u)=u e^{-a u}$ satisfies (8), $f(u)$ is increasing on $[0,1 / a], f(u)>0$ for $u \in(0,1 / a]$, and $f(u)$ attains its maximum $f_{\max }=(a e)^{-1}$ at $u=1 / a$. Since $f$ is continuous, there exists $r_{0}$ with $0<\delta_{0}<\sigma r_{0}<r_{0} \leq 1 / a$ such that $f\left(r_{0}\right)=r_{0} e^{-a r_{0}}=C_{r_{0}}>$ $\delta_{0} e^{-a \delta_{0}}=f\left(\delta_{0}\right)>0$. Thus

$$
\begin{aligned}
m\left(r_{0}\right) & =\min _{\sigma r_{0} \leq u \leq r_{0}} f(u)=f\left(\sigma r_{0}\right)=\sigma r_{0} e^{-a \sigma r_{0}}=\sigma r_{0}^{1-\sigma} r_{0}^{\sigma} e^{-a \sigma r_{0}} \\
& =\sigma r_{0}^{1-\sigma}\left\{f\left(r_{0}\right)\right\}^{\sigma} \geq \sigma r_{0}^{1-\sigma} C_{r_{0}}^{\sigma}>0 .
\end{aligned}
$$

Set

$$
X_{r_{0}}=\left\{\eta \in C_{T}\left(\mathbb{R}^{+}\right):\|\eta\|<r_{0}\right\} .
$$

Then $\Phi: P_{2} \cap \bar{X}_{r_{0}} \rightarrow P_{2}$ is completely continuous and satisfies all conditions of Theorem 5. Thus there exists $\mu>0$ such that (17) holds and $\|\eta\|=r_{0}$.

Acknowledgements. This project was supported by the Natural Science Foundation of Shanxi Province (2008011002-1), Shanxi Datong University, the Development Foundation of Higher Education Department of Shanxi Province (200613041, 200713028) and the Science and Technology Bureau of Datong City.

\section{References}

[1] S. S. Cheng and G. Zhang, Existence of positive periodic solutions for non-autonomous functional differential equations, Electron. J. Differential Equations 2001, no. 59, 8 pp.

[2] D. J. Guo, Fixed points and eigenelements of a class of concave and convex operators, Chinese Sci. Bull. 30 (1985), no. 15, 1132-1135 (in Chinese).

[3] D. J. Guo and V. Lakshmikantham, Nonlinear Problems in Abstract Cones, Academic Press, Orlando, FL, 1988. 
[4] W. S. Gurney, S. P. Blythe and R. M. Nisbet, Nicholson's blowflies revisited, Nature 287 (1980), 17-21.

[5] S. G. Kang, B. Shi and G. Zhang, Existence of three periodic positive solutions for a class of integral equations with parameters, J. Math. Anal. Appl. 323 (2006), 654-665.

[6] S. G. Kang, G. Zhang and S. S. Cheng, Periodic solutions of a class of integral equations, Topol. Methods Nonlinear Anal. 22 (2003), 245-252.

[7] H. Wang, Positive periodic solutions of functional differential equations, J. Differential Equations 202 (2004), 354-366.

[8] G. Zhang and S. S. Cheng, Positive periodic solutions of nonautonomous functional differential equations depending on a parameter, Abstract Appl. Anal. 7 (2002), 256269.

College of Mathematics and Computer Science

Shanxi Datong University

Datong, Shanxi 037009, P.R. China

E-mail: dtkangshugui@yahoo.com.cn

Department of Basic Sciences Naval Aeronautical Engineering Institute Yantai, Shandong 264001, P.R. China E-mail: baoshi781@sohu.com

Department of Mathematics

Tsing Hua University

Hsinchu, Taiwan 30043, R. O. China

E-mail: sscheng@math.nthu.edu.tw

Received 24.9.2008

and in final form 17.6.2009 